\title{
ESTILOS PARENTALES DISFUNCIONALES Y CONDUCTAS EMOCIONALES INADAPTADAS EN ADOLESCENTES DE UN COLEGIO DE LIMA SUR*
}

\section{Dysfunctional parenting styles and maladaptive emotional behaviors in adolescents of a school in South Lima}

Andrea Marina Canessa Cárdenas (Asociación de Hogares Nuevo Futuro Perú)**

Maria Gracia Lembcke Amayo (Colegio Alternativo Talentos, Trujillo - Perú)***

\begin{abstract}
Resumen
La presente investigación tiene como finalidad conocer la relación entre los estilos parentales disfuncionales percibidos y las conductas emocionales inadaptadas en estudiantes de 2do, 3ro y 4to de secundaria de un colegio de Lima Sur. La muestra estuvo conformada por 284 estudiantes de ambos sexos con edades que oscilan entre los 13 y 15 años. La investigación fue de tipo no experimental con un diseño correlacional. Para la medición de las variables se utilizaron dos escalas; la primera fue la Escala de estilos parentales disfuncionales (MOPS) y la segunda fue la Escala de evaluación de la conducta emocional inadaptada para niños y adolescentes (API-N). El resultado obtenido confirmó la hipótesis general y demostró la existencia de una correlación altamente significativa entre los estilos parentales disfuncionales del padre y las conductas emocionales inadaptadas de los adolescentes (.344**), así como la relación entre los estilos parentales disfuncionales de la madre y las conductas emocionales inadaptadas (.385**). Asimismo, se encontró relación significativa entre las diferentes dimensiones de las variables, a excepción de los estilos de indiferencia tanto del padre como la madre con la escala autonómica, y del estilo de indiferencia de la madre con la escala emocional.
\end{abstract}

Palabras claves: Estilos parentales disfuncionales, conductas emocionales inadaptadas, adolescentes.

\begin{abstract}
The aim of this research is to determine the relationship existing between the dysfunctional parenting styles perceived and maladaptive emotional behaviors in $2 n d, 3 r d$, and 4 th year students of a secondary school in South Lima. The sample consisted of 284 students of both sexes, between 13 and 15 years old. It was a non-experimental type research with a correlational design. To measure the variables, two scales were used: the first one was the Measure of parental styles (MOPS), and the second was the Rating scale for maladaptive emotional behavior of children and adolescents (API-N). The result obtained confirmed the general hypothesis, showing the existence of a highly significant correlation between the dysfunctional parenting style of the father and the maladaptive emotional behavior of the adolescents (.344**), as well as between the dysfunctional parenting style of the mother and the maladaptive emotional behaviors (.385**). Moreover, a significant correlation was found among the various dimensions of the variables, except for the indifference style of both the father and the mother with the autonomic scale, and of the mother's indifference style with the emotional scale.
\end{abstract}

Keywords: dysfunctional parenting styles, maladaptive emotional behaviors, adolescents.

\footnotetext{
* Artículo basado en la tesis de Licenciatura sustentada por las autoras.

** Psicóloga. andrea.canessa.17@gmail.com ORCID: https://orcid.org/0000-0001-8336-8369

*** Psicóloga.mg.lembckea@gmail.com ORCID: https://orcid.org/0000-0002-5717-6184
} 


\section{INTRODUCCIÓN}

La presente investigación surge a raíz de la necesidad de conocer una de las posibles causas del desarrollo de sintomatología inadaptada en los adolescentes. Según estadísticas actuales de la OMS, se calcula que, en el mundo, aproximadamente entre el $10 \%$ y $20 \%$ de los adolescentes, presentan problemas de salud mental (OMS, 2018). Se sabe también que existen muchos factores que pueden influenciar en el surgimiento de conductas emocionales inadaptadas en los adolescentes, uno de ellos está relacionado al factor familiar.

El rol fundamental de los padres es permitir que sus hijos adquieran la madurez biológica, psicológica y social necesaria para que se desempeñen adecuadamente dentro del ámbito familiar y social (Derbez y col., 2004). Cuando esto no sucede, pueden aparecer una serie de consecuencias a nivel de salud mental. Según diversas investigaciones como las de Alcázar, Parada-Navas, y Rosa-Alcázar (2014) y las de Taboada, Ezpeleta, y de la Osa (1998), existe relación entre los estilos parentales y los trastornos internalizantes en los niños, de estos trastornos los más relevantes son los siguientes: ansiedad y depresión (como se cita en Aguilar, Y., Raya, A., Pino, M. y Herruzo, J., 2019).

\section{MARCO TEÓRICO}

\section{Estilos parentales}

El estilo parental es el conjunto de estrategias utilizadas por los progenitores para conseguir sus objetivos respecto a la crianza de los hijos y toma como base dos factores: afecto y control (Moral, 2012). Como afirman Matalinares, Raymundo y Baca (2014), las estrategias utilizadas por los progenitores involucran una serie de comportamientos y actitudes hacia el menor que generan determinado clima emocional y que ejercen una influencia dentro de esa organización.

Diana Baumrind (1966), publicó un estudio titulado "Efectos del control parental autoritativo en el comportamiento de los niños", en esta investigación publicó tres prototipos de estilos parentales: autoritativo, autoritario y permisivo. Estos estilos parentales se basan en el grado de control que utilizan los padres para guiar y orientar la crianza de sus hijos, generando, cada uno de ellos, diversas consecuencias a nivel comportamental.

A raíz de los trabajos de Baumrind el concepto de estilos parentales fue consolidándose dentro de las variables psicológicas y diversos autores empezaron a desarrollarla, entre ellos, Maccoby y Martin, quienes en 1983 realizaron una variación del modelo de Baumrind y añadieron una nueva dimensión para la medición parental, obteniendo así un modelo de dos dimensiones: afecto/comunicación y control/ exigencia. Como consecuencia de esto, se propuso la existencia de un nuevo estilo parental: el estilo parental indulgente (Torío, Peña y Rodríguez, 2008).

De acuerdo a la teoría propuesta por Maccoby y Martin en 1983, el estilo autoritario-recíproco o también conocido como autoritativo presenta niveles acordes tanto de control como de afecto, mientras que el estilo autoritario se caracteriza por un alto nivel en la variable control y un bajo nivel en la variable afecto. En el estilo de crianza permisivo-indulgente el nivel de afecto es alto mientras que el nivel de exigencia es bajo y el estilo permisivo-negligente presenta ambas variables en un nivel bajo (Raya, 2008).

En trabajos posteriores, Baumrind (1996), manifestó que cada una de las dimensiones de los estilos parentales está compuesta por una serie de características. Así, la implicación afectiva está compuesta por la afabilidad, la comunicación, el apego y la reciprocidad; mientras que el control y exigencia está compuesto por la supervisión, la disciplina consistente y la confrontación (como se citó en Raya 2008).

Siguiendo en la línea de estilos parentales; Parker, Tupling y Brown (1979), publicaron una investigación en la que propusieron un nuevo instrumento para evaluar los lazos parentales, titulado "A parental bonding instrument". En esta investigación, los autores propusieron la existencia de cuatro estilos parentales basados en la bipolaridad de dos dimensiones: cuidado y sobreprotección. De acuerdo a estas dimensiones, los autores encontraron la existencia de cuatro estilos parentales: Cuidado óptimo, compulsión afectiva, 
control sin afecto y negligente o descuidado (como se citó en Asili y Pinzón, 2003).

En conclusión, existen cuatro estilos parentales de los cuales solo uno es funcional: el estilo parental autoritativo o de cuidado óptimo. Este estilo, también llamado democrático, es aquel en el que se brinda, no solo un espacio de crianza afectivo y cálido sino también un espacio donde el control y la disciplina no son invasivos ni restrictivos. Por el contrario, están basados en la reciprocidad y la comunicación (Torío, Peña y Rodríguez, 2008). A continuación, se muestra un cuadro con los tipos de vínculos establecidos por Parker y colaboradores.

\begin{tabular}{l|l|l|}
\multicolumn{2}{c}{ Alta sobreprotección } \\
\cline { 2 - 3 } Bajo cuidado & $\begin{array}{l}\text { Control } \\
\text { sin afecto }\end{array}$ & $\begin{array}{l}\text { Constricción } \\
\text { cariñosa }\end{array}$ \\
\cline { 2 - 3 } Alto cuidado & $\begin{array}{l}\text { Vínculo } \\
\text { Ausente }\end{array}$ & $\begin{array}{l}\text { Vínculo } \\
\text { Óptimo }\end{array}$ \\
\hline
\end{tabular}

Baja sobreprotección

Figura 1. Tipos de vínculos arrojados por el P.B.I. (Parental Bonding Instrument). Realizado por Melis, Francisca, Dávila, María de los Ángeles, Ormeño, Verónica, Vera, Valeria, Greppi, Claudio, \& Gloger, Sergio. (2001).

Los estilos de crianza o estilos parentales influyen, ya sea de manera positiva o negativa a nivel de clima emocional dentro del desarrollo de los niños (Gonzáles Vigil, 2008). Según estudios de Baumrind (1966), el estilo parental autoritativo genera consecuencias positivas a nivel de socialización y de independencia en los niños y adolescentes. De igual manera, está relacionado a la responsabilidad social en los chicos y en el caso de las mujeres, está relacionado con los altos logros (Baumrind, 1971). A diferencia de este estilo, los otros tres estilos están relacionados con posibles consecuencias a nivel de salud mental en los individuos (Parker et al., 1997).

\section{ESTILOS PARENTALES DISFUNCIONALES}

De acuerdo a las dimensiones propuestas por Parker et al. (1997), los estilos parentales disfuncionales, están caracterizados ya sea por un control inadecuado (abuso), por una protección excesiva (sobreprotección) o por un fallo en los cuidados parentales (negligente). Estos estilos parentales disfuncionales pueden ocasionar una serie de conflictos a lo largo de la vida del ser humano y están relacionados con problemas a nivel de interacción, así como con la aparición y desarrollo de enfermedades psiquiátricas (Matalinares et al., 2014).

Barudy y Dantagnan (2005), señalan que los padres que presentan un estilo parental incompetente se vinculan con sus hijos a través del apego inseguro, ya que no presentan los cuidados adecuados que se requieren para establecer un apego seguro. De igual manera, ambos autores afirman que, asegurando vínculos afectivos de calidad en la relación con los hijos, se puede lograr la salud mental de los mismos.

\section{Estilo parental de abuso}

Se caracteriza por las escasas manifestaciones de afecto y por el rechazo hacia el menor, así como por un control exagerado, en el que el uso de castigos y amenazas es constante (Barudy y Dantagnan, 2005). Este estilo se encuentra en el polo de las dos variables en las que se basan los estilos parentales y presenta consecuencias a nivel emocional y cognitivo, puesto que, según Lamborn, Mounts, Stenberg y Dornbusch (1991), si bien los adolescentes criados bajo este estilo parental presentan un alto nivel de conformidad $\mathrm{y}$ obediencia, presentan un autoconcepto pobre en comparación con otros adolescentes.

\section{Estilo parental permisivo o de sobreprotección}

Parker, Tupling y Brown (1979), exponen que este estilo está caracterizado por un excesivo cuidado representado por la complacencia ante las necesidades afectivas de los menores, acompañado de un control mínimo o imperceptible. Diana Baumrind (1966), señala que estos padres se caracterizan por aceptar todo tipo de deseos y acciones de sus hijos, evitando utilizar sanciones o correcciones claras y dejando de lado todo tipo de normas.

De acuerdo a Torío, Peña y Rodríguez (2008), los adolescentes que han sido educados bajo un 
estilo parental permisivo-indulgente suelen ser más originales y creativos, sin embargo, se caracterizan por estar más propensos a tener dificultades para regularse y controlar la expresión de sus emociones, así mismo, tienen mayores dificultades para mostrarse responsables y presentan resultados más bajos a nivel académico en comparación con otros adolescentes.

\section{Estilo parental negligente}

El estilo parental negligente se caracteriza por la poca capacidad de los padres para brindar los cuidados $\mathrm{y}$ atenciones necesarios para el desarrollo de sus hijos, así como por la poca capacidad para ejercer control sobre ellos. Dentro de este estilo parental no existen normas ni límites claros, de igual manera, no existe una comunicación afectiva, por el contrario, la carencia afectiva es base de este estilo de crianza (Barudy y Dantagnan, 2005). Los niños y adolescentes que han sido educados bajo un estilo parental negligente presentan resultados más bajos a nivel de autoestima, logros, autonomía y responsabilidad en comparación con otros individuos (Torío, Peña y Rodríguez, 2008).

\section{ESTILOS PARENTALES Y FAMILIA}

Estévez, Jiménez y Musitu (2008) señalan que la familia tiene como función principal generar el bienestar psicológico de sus miembros a través de las relaciones basadas en el afecto y el soporte que les brindan (como se citó en Morales, 2018). Según Moraleda, durante la infancia, la familia es el grupo referente más importante puesto que es el lugar donde el menor puede establecer interacciones con otros (Aguirre, 1994) y, de acuerdo a la calidad del vínculo que se haya establecido en esta etapa, se generará una continuación en la adolescencia, influenciando así, en el desarrollo adecuado de relaciones afectivas con sus progenitores (Fuentes, Motrico y Bersabé, 2003).

En la familia, es de vital importancia brindar a los hijos un ambiente de cuidado, afecto y comunicación asertiva, pues es este espacio el que será el modelo de las interacciones de los adolescentes, y de igual manera, influirá en el bienestar de la salud mental de los mismos (Huamán, 2016). De acuerdo a Paul A. Hauck, los errores cometidos por los padres durante la crianza de los hijos producen una serie de consecuencias en la educación de los mismos. Para este autor, cuando los padres utilizan modelos de rigidez extrema o de amabilidad exagerada pueden ocasionar el desarrollo de ciertos trastornos como la depresión, el trastorno obsesivo compulsivo o la neurosis por angustia (Ellis y Grieger, 2003).

\section{PSICOPATOLOGÍA INFANTIL Y DEL ADOLESCENTE: TRASTORNOS EMOCIONALES}

Un trastorno mental es un síndrome caracterizado por una alteración clínicamente significativa del estado cognitivo, la regulación emocional o el comportamiento del individuo. Este síndrome refleja una disfunción de los procesos psicológicos, biológicos o del desarrollo que subyacen en su función mental. Habitualmente, los trastornos mentales van asociados a un estrés significativo o a una discapacidad, ya sea social, laboral o de otras actividades importantes. [American Psychiatric Association (APA), 2015, p. 20].

En la actualidad, el Manual Diagnóstico de los Trastornos mentales incluye también a la psicopatología infantil y del adolescente. Sin embargo, antiguamente este campo había sido descuidado ya que el diagnóstico y tratamiento estaba enfocado en la clínica adulta (Caballo y Simón, 2002). Asimismo, es sabido que existen diferencias tanto en sus manifestaciones como en su evaluación y tratamiento, lo cual requiere un abordaje teórico específico.

No obstante, no existe una definición específica de patología infantil. Por esta razón se sigue utilizando como base las definiciones propuestas en el DSM (en todas sus versiones). Aun así; es necesario recalcar que, como señala Massick, en la psicopatología infantil y del adolescente deben tomarse en cuenta tres variables: en primer lugar, entender que el niño y el adolescente presenta características propias que influyen en el curso de la enfermedad; en segundo lugar, los antecedentes familiares y contextuales influyen también en el desarrollo de la enfermedad, y por último, el niño y el adolescente tienen como característica el dinamismo y el cambio, por lo que los resultados en un momento pueden diferir posteriormente (como se citó en Caballo y Simón, 2002). Es importante mencionar también que, a nivel de causas para el desarrollo de la conducta anormal, no existe un solo factor que lo determine, 
sino que es producto de la presencia de una serie de factores tanto biológicos como sociales.

Los diferentes trastornos emocionales tienen manifestaciones observables a través de las cuales se puede realizar un posible diagnóstico. Es importante mencionar que, como exponen Mash y Terdal, la evaluación conductual del niño pretende ser "una serie de estrategias de solución de problemas intentando comprender tanto a los niños con problemas y sin problemas como a sus sistemas sociales, incluyendo sus familias y grupos de iguales" (como se citó en Caballo y Simón, 2002).

\section{TRASTORNOS EMOCIONALES Y SU CONCEPCIÓN CONDUCTUAL}

Ahora bien, bajo un enfoque cognitivo conductual, los trastornos emocionales o mentales reciben la denominación de conductas emocionales inadaptadas (Parasi, 2015). Entonces, partiendo como base de este marco teórico, los problemas o desórdenes emocionales, deben ser medidos bajo su manifestación conductual. Según Anicama, estos desórdenes se manifiestan a través de cinco niveles conductuales, entre los que se encuentran los niveles autonómico, emocional, motor, social y cognitivo (como se citó en Parasi, 2015).

\section{NIVELES DE CONDUCTAS EMOCIONALES INADAPTADA}

Como se mencionó anteriormente, las conductas emocionales inadaptadas se expresan a través de cinco niveles conductuales (autonómico, emocional, motor, social y cognitivo), y cada uno de estos presenta una serie de manifestaciones observables.

A continuación, se presenta una figura basada en la teoría de Anicama donde se resumen las expresiones conductuales de cada uno de los componentes y posteriormente se desarrollará cada uno de los mismos.

Niveles

E1

E2

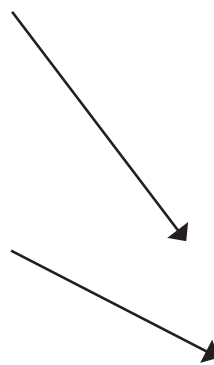

E3

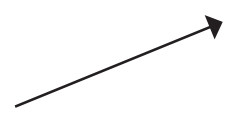

Variables

intervinientes:

- Fisiológicas

- Sociales

- Psicológicas

\section{Expresión conductual}

\section{(1) Autonómico:}

Palpitaciones, taquicardia, dolor de cabeza, síntomas somáticos, etc.

(2) Emocionales:

Irritabilidad, sentimiento de ansiedad, tristeza, llanto, etc.

(3) Motoras:

Conducta de evitación, reducción de la actividad regular, retardo psicomotor, etc.

(4) Sociales:

Mínima interacción, aislamiento, conductas antisociales, etc.

\section{(5) Cognitivas:}

Pensamientos persistentes, expectativas negativas, autocrítica acentuada, baja autoestima, etc.

Figura 2. Manifestaciones conductuales de desórdenes emocionales. Elaborado por Anicama (1979, 1987, 1993, 2010). Rescatado por Parasi (2015). 


\section{Nivel Autonómico}

De acuerdo al cuadro mostrado, se evidencia que este nivel hace referencia a toda manifestación somática surgida durante la presencia de un desorden emocional. Entre estas, destacan: las palpitaciones, la taquicardia, los dolores de cabeza, la pérdida del apetito, las alteraciones del sueño, etc (Anicama, citado por Parasi, 2015).

El nivel autonómico está relacionado con la somatización. Esta se caracteriza por la "expresión orgánica de un deterioro psicológico (APA. Diccionario Conciso de Psicología, 2010).

\section{Nivel Emocional}

Como se sabe, la emoción es una reacción compleja que incluye manifestaciones fisiológicas y conductuales (APA. Diccionario Conciso de Psicología). Este nivel corresponde al de las expresiones conductuales relacionadas a los afectos negativos, entre las que se encuentran el llanto, el sentimiento de angustia, la labilidad emocional, la falta de respuesta de alegría, etc (Anicama, citado por Parasi, 2015).

\section{Nivel Motor}

El término motor en la psicología está relacionado a todo aquello que involucre los movimientos musculares (APA. Diccionario Conciso de Psicología, 2010). Por tanto, este nivel hace referencia a toda aquella manifestación observable relacionada con las destrezas que involucren movimiento a nivel muscular.

En este nivel se encuentran manifestaciones tales como la reducción de respuesta psicomotora (habla o marcha disminuida), la tensión muscular, el tartamudeo o el temblor de alguna zona del cuerpo (Anicama, citado por Parasi, 2015).

\section{Nivel Social}

A nivel social las manifestaciones están relacionadas a toda interacción, ya sea asocial o antisocial. Entre ellas se encuentran el aislamiento, la agresión, la violencia, la escasa participación a nivel grupal, etc.

De acuerdo a Del Barrio y Carrasco (2016), en este nivel pueden verse afectas las interacciones con los miembros de la familia. De igual manera, puede presentarse aislamiento o desinterés.

\section{Nivel Cognitivo}

Según el Diccionario de la Real Academia Española de la Lengua (2001), lo cognitivo hace referencia a todo tipo de conocimiento o acto de conocer, el cual está relacionado con la razón y el pensar.

De acuerdo a Anicama (como se citó en Parasi, 2015), las manifestaciones conductuales de este nivel están relacionadas a los pensamientos. Entre estos últimos destacan la percepción e ideas de fracaso, las expectativas negativas, los pensamientos persistentes, entre otros.

Debido a la escasa cantidad de investigaciones estadísticas relacionadas a las posibles causas del desarrollo de problemas emocionales en los adolescentes de nuestra sociedad, y menos aún, de investigaciones directamente relacionadas a los estilos parentales, surgió el interés de investigar la relación existente entre los estilos parentales disfuncionales y las conductas emocionales inadaptadas en los adolescentes. Por esta razón, formulamos la siguiente pregunta: ¿Existe relación significativa entre los estilos parentales disfuncionales del padre y de la madre y las conductas emocionales inadaptadas en adolescentes de 2do, 3ro y 4to grado de nivel secundario de un colegio de Lima Sur?

\section{MÉTODO}

\section{Tipo y diseño de la investigación}

Investigación de tipo cuantitativo, diseño descriptivo y correlacional.

\section{Participantes}

La muestra estuvo conformada por 284 adolescentes varones y mujeres que se encontraban cursando 2ro, 3to y 4to año grado de nivel secundario dentro de la institución y cuyas edades oscilaban entre 13 y 15 años.

\section{Instrumentos}

Se utilizó la Escala de Estilos Parentales 
Disfuncionales (MOPS), creada por Parker, Roussos, Hadzi-Pavlovic, Mitchell, Wilhelm y Austin (1997) y adaptada al Perú por Matalinares, Raymundo y Baca en el año 2014 obteniendo una confiabilidad de 0.87 para la variable padre y 0.80 para la variable madre.

Asimismo, se utilizó la Escala de evaluación de conducta emocional inadaptada (API-N), elaborada en Lima en el año 2015 por Claudia Fabiana Parasi Alarcón con el objetivo de identificar la presencia de conductas emocionales inadaptadas, así como sus manifestaciones a nivel de sus componentes (autonómico, emocional, conductual, cognitivo y escala motora) en niños y adolescentes entre 8 y 15 años. La presente escala presenta una confiabilidad de 0.918 según alfa de Cronbach.

\section{Procedimiento}

Se solicitó a las autoridades de la Institución Educativa el permiso correspondiente para realizar la investigación.

Posteriormente, se presentaron los asentimientos informados y se continuó con la aplicación de los instrumentos a los estudiantes de los grados seleccionados.

Una vez aplicadas las pruebas, se procedió a la corrección manual que llevó a la eliminación de aproximadamente 66 pruebas.

Finalmente, se continuó con la interpretación de los baremos y al vaciado de datos y se realizó el procesamiento de los datos obtenidos a través del programa SPSS 25.

\section{RESULTADOS}

A continuación, se presentarán los resultados obtenidos durante la investigación.
Tabla 1

Prueba de normalidad según Kolmogorov-Smirnov

\begin{tabular}{lcc}
\hline & Estadístico & Sig. \\
\hline Escala Autonómica & .108 & $.000^{*}$ \\
Escala Emocional & .062 & $.010^{*}$ \\
Escala Social & .066 & $.004^{*}$ \\
Escala Motora & .094 & $.000^{*}$ \\
Escala Conductual & .120 & $.000^{*}$ \\
API-N Total & .059 & $.019^{*}$ \\
Abuso Padre & .171 & $.000^{*}$ \\
Sobreprotección Padre & .249 & $.000^{*}$ \\
Indiferencia Padre & .188 & $.000^{*}$ \\
Padre Total & .165 & $.000^{*}$ \\
Abuso Madre & .224 & $.000^{*}$ \\
Sobreprotección Madre & .215 & $.000^{*}$ \\
Indiferencia Madre & .211 & $.000^{*}$ \\
Madre Total & .165 & $.000^{*}$ \\
\hline
\end{tabular}

*corrección de significación de Lilliefors

En la tabla 1, se observa que no existe una distribución normal entre las variables, así como tampoco entre las dimensiones de cada variable, razón por la cual se procedió a utilizar pruebas no paramétricas para la demostración de la hipótesis estadística.

A continuación, se muestra la tabla de correlación según Spearman, en la que se puede observar una correlación positiva entre cada uno de los estilos parentales con el total de las conductas emocionales inadaptadas.

Tabla 2

Medidas de correlación según Spearman

\begin{tabular}{|c|c|c|c|c|c|c|}
\hline & Autonómico & Emocional & Motor & Social & Cognitivo & API-N \\
\hline Abuso Padre & $.160 * *$ & $.166^{* *}$ & $.160 * *$ & $.233 * *$ & $.329 * *$ & $.279 * *$ \\
\hline Sobreprotección Padre & $.230 * *$ & $.285 * *$ & $.253 * *$ & $.287 * *$ & $.336 * *$ & $.377 * *$ \\
\hline Indiferencia Padre & .079 & $.157 * *$ & $.157 * *$ & $.218 *$ & $.272 * *$ & $.244 * *$ \\
\hline Padre Total & $.178^{* * *}$ & $.214 * *$ & $.207 * *$ & $.294 *$ & $.385 * *$ & $.344 * *$ \\
\hline Abuso Madre & $.220 *$ & $.289 * *$ & $.197 *$ & $.238 * *$ & $.347 * *$ & $.348 * *$ \\
\hline Sobreprotección Madre & $.274 * *$ & $.329 *$ & $.218 * *$ & $.333 * *$ & $.291 * *$ & $.393 * *$ \\
\hline Indiferencia Madre & .022 & .112 & $.142 * *$ & $.205 * *$ & $.150 *$ & $.166^{* *}$ \\
\hline Madre Total & $.223 * *$ & $.300 * *$ & $.236 *$ & $.338 * *$ & $.336 * *$ & $.385^{* * *}$ \\
\hline
\end{tabular}


En la tabla 2, se observa que existen correlaciones entre las áreas de los estilos parentales disfuncionales percibidos por los estudiantes y las conductas emocionales inadaptadas de los mismos. Asimismo, se observa que la variable de Estilos Parentales Disfuncionales Total del Padre tiene una mayor correlación significativa con la Escala Cognitiva, mientras que la variable Estilos Parentales Disfuncionales Total de la Madre tiene una mayor correlación con el total de las Conductas Emocionales Inadaptadas.

\section{DISCUSIÓN DE RESULTADOS}

$\mathrm{Al}$ realizar los análisis estadísticos para establecer la correlación entre los estilos parentales disfuncionales y las conductas emocionales inadaptadas en adolescentes de 2do, 3ro y 4to grado de nivel secundario de un colegio de Lima Sur; se encontró que existe una correlación positiva entre ambas variables. Esto último demostraría la influencia que produce el estilo de crianza disfuncional recibido durante la infancia, niñez y adolescencia en el desarrollo de conductas emocionales no adecuadas en los adolescentes evaluados.

Los resultados obtenidos refuerzan los estudios previos realizados en diversos países por otros autores (Aguilar-Yamuza, Raya, Pino y Herruzo, 2018; Fuentes, García, Gracia y Alarcón, 2015; Vallejo, Osorno y Madaziego, 2008; Rojas, 2016; entre otros). En estos se señala la correlación positiva entre los estilos parentales disfuncionales y la salud mental. Asimismo, confirma lo expresado por autores como Barudy y Dantagnan (2005), quienes afirman que el entorno cumple un rol importante en la modulación y formación de los niños y niñas y que la crianza parental no adecuada acompañada de malos tratos produce daños graves.

Respecto a la relación entre el estilo parental de abuso del padre y las conductas emocionales inadaptadas, se encontró que existe una correlación positiva y significativa con cada una de las dimensiones de las conductas emocionales inadaptadas de los adolescentes evaluados. De acuerdo al estudio realizado por Lamborn, Mounts, Steinberg y Dornbusch (1991), los adolescentes que han tenido padres autoritarios tienen percepciones de sí mismos más bajas en comparación con los adolescentes que han sido criados bajo otros estilos parentales.

En cuanto a la relación entre el estilo parental de sobreprotección del padre y las conductas emocionales inadaptadas, se encontró que existe relación positiva con cada una de las dimensiones de la variable dependiente. Capano y Ubach (2013), afirman que los adolescentes que han vivido bajo un estilo permisivo y sobreprotector generalmente presentan problemas de confianza y seguridad, y suelen tener baja autoestima.

En relación al estilo parental negligente del padre, se encontró que existe una correlación positiva con todas las dimensiones de las conductas emocionales inadaptadas de los adolescentes, a excepción de la dimensión autonómica. Según Sánchez y Hernández, el rechazo y poca presencia del padre produce mayores efectos negativos en el menor en comparación con el rechazo de la madre, produciéndose baja autoestima y falta de motivación (como se citó en Asili y Pinzón, 2003).

En cuanto al análisis estadístico respecto a los estilos parentales percibidos de la madre, los resultados son idénticos a los obtenidos respecto al padre. Se encontró que a nivel total existe una correlación positiva entre los estilos parentales disfuncionales y el desarrollo de conductas emocionales inadaptadas en los adolescentes en cada una de las dimensiones evaluadas (autonómica, emocional, social, motora y cognitiva). Como expuso Bowlby en 1954, los cuidados brindados por las figuras parentales durante los primeros años de vida ejercen una influencia fundamental en la salud mental futura de los seres humanos.

A nivel de estilo parental de abuso de la Madre, se encontró una correlación positiva con las conductas emocionales inadaptadas en los adolescentes en todas las dimensiones de la misma (autonómico, emocional, motor, social y cognitivo). Según Baumrind, los niños $\mathrm{y}$ adolescentes que han vivido dentro de una familia donde el estilo parental preponderante ha sido el autoritario, sufren consecuencias negativas mayores. De acuerdo a sus estudios, los adolescentes criados bajo este estilo parental son más inseguros, menos 
felices y más hostiles (como se citó en Kaufmann et al., 2000).

Respecto al estilo parental de sobreprotección de la madre, la correlación con las conductas emocionales inadaptadas en los adolescentes es altamente positiva en cada una de las dimensiones. Torío, Peña y Rodríguez (2008) afirman que los adolescentes que provienen de un hogar donde la permisividad es la base de la crianza presentan consecuencias a nivel social, conductual y emocional; caracterizándose por una carente competencia social, dificultad para controlar impulsos, dependencia y escasa motivación.

En cuanto al estilo parental de indiferencia de la madre, se encontró una correlación significativa con la presencia de conductas emocionales inadaptadas solo en 3 dimensiones, a excepción del nivel autonómico y del nivel emocional. Se corroboró la información expuesta anteriormente donde se señala que, a nivel de indiferencia, es más significativa en el adolescente la poca presencia del padre y su poco interés sobre él, que el rechazo percibido por la madre (Asili y Pinzón, 2003).

De acuerdo a los resultados expuestos se demostró la hipótesis general de la presente investigación, así como cada una de las hipótesis específicas propuestas. Sin embargo, debido a que en la prueba utilizada las conductas emocionales inadaptadas pueden ser divididas en subescalas, se encontró una relación inversa entre el estilo de indiferencia del padre con la escala autonómica de las conductas emocionales inadaptadas; de igual forma, se encontró relación inversa entre el estilo de indiferencia de la madre y dos de las subescalas de las conductas emocionales inadaptadas: autonómica y emocional.

A partir de la investigación realizada y de los resultados obtenidos se puede concluir que existe una correlación significativa entre los estilos parentales disfuncionales, donde se encuentran los estilos parentales de abuso, sobreprotección y negligencia tanto del padre como de la madre- y la aparición o desarrollo de conductas emocionales inadaptadas en los adolescentes de 2do, 3ro y 4to grado de nivel secundario de un colegio de Lima Sur.

\section{CONCLUSIONES}

Se determinó una relación directa entre los estilos parentales disfuncionales del padre y de la madre a nivel general, y las conductas emocionales inadaptadas en los adolescentes de 2do, 3ro y 4to grado de nivel secundario de un colegio de Lima Sur.

En cuanto a los estilos parentales del padre, se encontró una relación directa entre los tres estilos parentales y el total de las dimensiones de conducta emocional inadaptada en los adolescentes de 2do, 3ro y 4to grado de nivel secundario de un colegio de Lima Sur. Sin embargo, en cuanto a la subescala autonómica de las conductas emocionales inadaptadas, se encontró una relación inversa con el estilo parental de indiferencia.

De igual forma, se precisó una relación directa entre los tres estilos parentales de la madre y el total de las dimensiones de conducta emocional inadaptada en los adolescentes de 2do, 3ro y 4to grado de nivel secundario de un colegio de Lima Sur. Sin embargo, existe una relación inversa entre el estilo parental de indiferencia y dos de las subescalas de la conducta emocional inadaptada en los estudiantes, siendo estas la escala autonómica y la escala emocional.

De acuerdo a lo encontrado, es necesario continuar la elaboración de investigaciones que correlacionen las variables utilizadas en el presente trabajo en diferentes colegios de Lima y de provincias. De igual manera, contrastar otras posibles variables, como nivel socioeconómico o grupos de edades. De igual forma, es necesario proponer investigaciones que correlacionen las variables apego o estilos parentales con variables netamente educativas, para así observar las posibles consecuencias a nivel académico, ya que en nuestro país los resultados a nivel educativo están por debajo de la media y se necesita un análisis detallado de las posibles causas.

De igual forma, es necesario establecer programas de intervención en la escuela que incluyan no solo a los niños, niñas y adolescentes, sino también a los padres y a los maestros, para que obtengan información clara y adecuada sobre la importancia de los estilos de crianza, así como estrategias directas de intervención. 


\section{REFERENCIAS}

Acosta, M. (08 de junio de 2018). Depresión: cuando vivir se convierte en una tarea insoportable. $E l$ comercio. Recuperado de https://elcomercio.pe/ lima/sucesos/vivir-convierte-tarea-insoportablenoticia-492188

Aguilar-Yamuza, B., Raya-Trenas, A. F., Pino-Osuna, M. J., y Herruzo-Cabrera, J. (2019). Relación entre el estilo de crianza parental y la depresión y ansiedad en niños entre 3 y 13 años. Revista de Psicología Clínica con Niños y Adolescentes. 6(1), 36-43. Recuperado de https://www.google.com/ search?q=aguilar-yamuza $+2019 \&$ oq=aguilar-ya muza+2019\&aqs $=$ chrome..69i57.38981j0j7\&sou rceid $=$ chrome $\&$ ie $=$ UTF- 8

Aguirre, A. (1994). Psicología de la Adolescencia. México: Alfaomega Grupo Editor.

American Psychiatric Association (2010). APA: Diccionario Conciso de Psicología. México: Manual Moderno.

American Psychiatric Association (2015). Manual Diagnóstico y Estadístico de los Trastornos Mentales (5ta ed.; DSM-5). Madrid: Médica Panamericana.

Asili, N. y Pinzón, B. (2003). Relación entre estilos parentales, estilos de apego y bienestar psicológico. Psicología y Salud, 13(2), 215-225.

Barudy, J. y Dantagnan, M. (2005). Los buenos tratos a la infancia. España: Gedisa.

Baumrind, D. (1966). Effects of Authoritative Parental Control on Child Behavior. Child Development, 37(4), 887-907.

Baumrind, D. (1971). Current patterns of parental authority. Developmental Psychology, 4(1), 1-103. doi:10.1037/h0030372

Blum, R. W. y Rinehart, P. M. (1997). Reducing the risk: Connections that make a difference in the lives of youth. Recuperado de https://files.eric. ed.gov/fulltext/ED412459.pdf

Bowlby, J. (1954). Los cuidados maternos y la salud mental. Organización mundial de la salud. Recuperado de http://hist.library.paho.org/ English/SPUB/41545.pdf

Bowlby, J. (1989). Una base segura: aplicaciones clínicas de una teoría del apego. España: Paidós.
Caballo, E. y Simón, M. (2002). Manual de psicología clínica infantil y del adolescente: trastornos generales. Madrid: Pirámide.

Caballo, E. y Simón, M. (2007). Manual de psicología clínica infantil y del adolescente: trastornos específicos. Madrid: Pirámide.

Capano, Á, y Ubach, A. (2013). Estilos parentales, parentalidad positiva y formación de padres. Ciencias Psicológicas, 7(1), 83-95. Recuperado de http://www. scielo.edu.uy/scielo.php?script=sci_arttext\&pid=S168842212013000100008\&lng=es\&tlng=en.

Carrillo, S.(1999). Mary SalterAinsworth(1913-1999). Revista Latinoamericana de Psicología, 31(2), 383-386.

Cava, M. (2003). Comunicación familiar y bienestar psicosocial en adolescentes. Encuentros en Psicología Social, 1, 23-27.

Coleman, J. y Hendry,L. (2003). Psicología de la adolescencia. Madrid: Ediciones Morata, S.L.

Del Barrio y Carrasco (2016). Problemas conductuales y emocionales en la infancia y la adolescencia. Padres y maestros, 365, 55-61. doi: pym.i365. y2016.008

Derbéz et. al. (2004). La dinámica de la familia. Revista Psicología, 68 (9), 1215-1219.

Duschinsky, R. y Solomon, J. (2017). Infant disorganized attachment: Clarifying levels of analysis. Clinical Child Psychology and Psychiatry 22(4), 524-538.

Echegoyen, J. (2011). Introducción a la Psicología UNED - Curso de acceso. Manual Fundamentos de la Psicología. Cap. 13. Los tratamientos psicológicos (Resumen). Recuperado de http://www.e- torredebabel.com/UnedParla/ Asignaturas/IntroduccionPsicologia/ ResumenManual- Capitulo13.htm

Ellis, A. y Grieger R. (2003). Manual de Terapia Racional Emotiva (9na ed.). Bilbao: Desclée de Brouwer.

Fuentes, M., García, F., Gracia, E. y Alarcón, A. (2015). Los estilos parentales de socialización y el ajuste psicológico. Un estudio en adolescentes españoles. Revista de Psicodidáctica, 20(1), 117-138.

Fuentes, M., Motrico, M. y Bersabé, R. (2003). Estrategias de socialización de los padres y conflictos entre padres e hijos en la adolescencia. Anuario de Psicología, 34(3), 385-400. 
García, A. (27 de junio del 2019). Preocupante situación de la Salud Mental: más de 6 millones de personas necesitan atención. Perú 21. Recuperado de https://peru21.pe/lima/preocupante-situacionsalud-mental-seis-millones-peruanos-necesitanatencion-480592.

González, L. y Méndez, L. (2006). Relación entre Autoestima, Depresión y Apego en adolescentes urbanos de la comuna de Concepción, Chile. Terapia psicológica, 24(1), 5-14.

González-Vigil, T. (2008). Relación entre percepción del apoyo parental a la autonomía e involucramiento parental con ansiedad en púberes (Tesis para la Licenciatura). Pontificia Universidad Católica del Perú. Lima, Perú.

Gozzer, M. y Santana. R. (2015). Sintomatología depresiva y estilos parentales en niños del colegio privado peruano canadiense de Chiclayo (tesis de Maestría). Universidad Privada Antonio Guillermo Urrelo, Cajamarca, Perú.

Hernández, R., Fernández, C. y Baptista, M. (2010). Metodología de la investigación, 5ta. Ed. México: Mc-Graw-Hill.

Huamán, K. (2016). Estilos parentales e indicadores de salud mental adolescente. Temática Psicológica, 12(1), 35-46.

Instituto Nacional de Salud Mental Honorio Delgado (2016). Maltrato infantil. Recuperado de http:// www.insm.gob.pe/oficinas/comunicaciones/ notasdeprensa/2016/033.html.

Kaufmann, D., Gesten, E., Santa Lucia, R., Salcedo, O., Rendina-Gobioff, G. \& Gadd, R. (2000). The relationship between parenting style and children's adjustment: The parent's perspective. Journal of Child and Family Studies, 9(2), 231245. doi: 10.1023/A:1009475122883

Lamb, M. E. (1980). Patterns of attachment: A psychological study of the strange situation. Mary D. Salter Ainsworth, Mary C. Blehar, Everett Waters, and Sally Wall. Hillsdale, N.J., Erlbaum, 1978. Infant Mental Health Journal, 1(1), 68-70. doi:10.1002/1097-0355(198021)1:1<68::aidimhj2280010110>3.0.co;2-3

Lamborn, S., Mounts, N., Steinberg, L. \& Dornbusch, S. (1991). Patterns of Competence and Adjustment among adolescents from Authoritative,
Authoritarian, Indulgent, and Neglectful Families. Child Development, 62(5), 1049 - 1065. doi: 10.1111/j.1467-8624.1991.tb01588.x

León, H. (2005). Estigma y enfermedad mental: un punto de vista histórico-social. Revista de Psiquiatría y Salud Mental Hermilio Valdizán, 6(1), 33-42. Recuperado de http://www.hhv. gob.pe/wp-content/uploads/Revista/2005/I/4ESTIGMA_Y_ENFERMEDAD_MENTAL.pdf

Main, M., \& Solomon, J. (1986). Discovery of an insecure-disorganized/disoriented attachment pattern. In T. B. Brazelton \& M. W. Yogman (Eds.), Affective development in infancy, 95-124.

Matalinares, M., Raymundo, O. y Baca, D. (2014). Escala de estilos parentales disfuncionales - MOPS - Perú.

Matalinares, M., Raymundo, O. y Baca, D. (2016). Escala de estilos parentales disfuncionales Manual- Perú.

Matalinares, M, \& Díaz, A. (2014). Influencia de los estilos parentales en la adicción al internet en alumnos de secundaria del Perú. Revista de investigación en psicología, 16(2), 195-220.

Melis, F., Dávila, M.A., Ormeño, V., Vera, V., Greppi, C., \& Gloger, S. (2001). Estandarización del P.B.I. (Parental Bonding Instrument), versión adaptada a la población entre 16 y 64 años del Gran Santiago. Revista chilena de neuro-psiquiatría, 39(2),132-139. https://dx.doi.org/10.4067/S071792272001000200005

Moneta, M. (2014). Apego y pérdida: redescubriendo a John Bowlby. Revista Chilena de Pediatría, 85(3), 265-268. DOI: 10.4067/S037041062014000300001

Moral, J. (2012). Prácticas parentales y percepción del rol en padres de adolescentes. Revista Mexicana de Investigación en Psicología, 5(1), 6-19. Recuperado de https://www.medigraphic.com/ pdfs/revmexinvpsi/mip-2013/mip131a.pdf

Morales, M. (2018). Estilos educativos parentales y adicción al internet en alumnos de secundaria en La Molina (Tesis de Maestría). UNIFE, Lima, Perú.

Musitu, G. y Cava, M. (2003). El rol del apoyo social en el ajuste de los adolescentes. Intervención Psicosocial, 12(2), 179-192.

Noller, P. \& Callan, V. (1991). The adolescent in the family. Londres: Routlege. 
Organización Mundial de la Salud. (2018). Salud mental del adolescente. Recuperado de https:// www.who.int/es/news-room/fact-sheets/detail/ adolescent-mental-health

Palacios, J., García, A. y Flores, E. (2010). Perfiles Emocionales en Madres Cuidadoras del Programa Nacional WawaWasi de Lima Metropolitana. Revista de Investigaciones Psicológicas de la Universidad Villarreal, 1(1). Recuperado de http://issuu.com/psicologiaunfv/docs/revista1/5

Parasi, C. (2015). Construcción y propiedades psicométricas de la escala de conducta emocional inadaptada en niños y adolescentes de 8 a 15 años de Lima Sur (tesis de pregrado). Universidad Autónoma del Perú, Lima, Perú.

Parker, G., Tupling, H. \& Brown, B. (1979). A parental Bonding instrument. British Journal of Medical Psychology, 52, 1-10. doi: 10.1111/j.2044-8341. 1979.tb02487.x

Parker, G., Roussos, J., Hadzi-Pavlovic, D., Mitchell, P., Wilhelm, K. \& Austin, M-P. (1997).The development of a refined measure of dysfunctional parenting and assessment of its relevance in patients with affective disorders. Psychological Medicine, 27(5), 1193-1203.

Ramírez, G. y Díaz, G. (2017). Estilos parentales percibidos y sintomatología depresiva en escolares de secundaria. CASUS, 2(2),119-126.

Raya, A. F. (2008). Estudio sobre los estilos educativos parentales y su relación con los trastornos de conducta en la infancia (Tesis doctoral). Universidad de Córdoba. Recuperado de http:// helvia.uco.es/xmlui/bitstream/ handle/10396/2351/abre_fichero. pdf?sequence

Real Academia Española. (2001). Diccionario de la lengua española (22 ed.). Consultado en https:// dle.rae.es

Rojas, L. (2016). Estilos parentales y salud mental en adolescentes de educación secundaria de las Instituciones Públicas de Casma (tesis de licenciatura). Universidad Cesar Vallejo, Lima, Perú.

Fecha de recepción: 8 de marzo 2020

Fecha de aceptación: 13 de abril 2020
Roldán, M.A.(1998, Mayo).Influencia de la estructura familiar en la construcción de la identidad de los adolescentes. Documento presentado en el VIII Congreso de INFAD, Pamplona, España.

Rygaard, N. (2008). El niño abandonado. España: Gedisa.

Salaverry, O. (2012). La piedra de la locura: inicios históricos de la salud mental. Revista Peruana de Medicina Experimental y Salud Pública, 29(1),143-48.

Sanchis, F. y Botella,L.(2007). Apego y sintomatología depresiva en la relación: su relación con los acontecimientos vitales. Revista de Psicoterapia, 18(72), 27-56.

Sarason, I. y Sarason, B. (2006). PSICOPATOLOGÍA. Psicología anormal: el problema de la conducta inadaptada. México: Pearson Educación.

Sausa, M. (03 de febrero del 2018). Menores ocupan el $70 \%$ de atenciones en salud mental. Perú 21. Recuperado de https://peru21.pe/peru/ cifras-salud-mental-peru-menores-ocupan-70atenciones-infografia-394376

Tam, J., Vera, G. y Oliveros, R. (2008). Tipos, métodos y estrategias de investigación. Pensamiento y acción, 5, 145-154.

Tamayo, M. (2003). El proceso de la Investigación Científica. México: Limusa.

Torío, S., Peña. J. y Rodríguez., M. (2008). Estilos educativos parentales. Revisión bibliográfica y reformulación teórica. Teoría de la educación, 20, $151-178$.

Torrel, M. y Delgado, M. (2014). Funcionamiento familiar y depresión en adolescentes de la I.E. Zarumilla - Tumbes, 2013. Ciencia y Desarrollo, 17- 48.

Vallejo, M., Osorno, R.y Madaziego, T. (2008). Estilos parentales y sintomatología depresiva en una muestra de adolescentes veracruzanos. Enseñanza e investigación, 13 (1) 91-105. 